Children vertically exposed to HIV demand care related to prophylaxis, follow-up in specialised service and food and nutrition. Breastmilk is the best way to feed a child and one of the most important interventions to reduce the risk of infant mortality. On the other hand, HIV positive women are discouraged from breastfeeding because of the risk of HIV transmission. Thus, in Brazil, children are guaranteed by law the integral and free supply of infant formula. Recognising the dependence of family care provided by feeding vertically exposed children to HIV, a research was developed to evaluate the family capacity to take care of the feeding of children exposed to HIV. A cross-sectional study, with the population of children born to caregivers exposed to HIV, aged zero to 24 months old, ongoing monitoring of health referral service in southern Brazil. Data collection performed with the application of the Scale to assess the capacity to care for children exposed to HIV and the Brazilian Scale of Food Insecurity. occurrence of three cases of breastfeeding supply, two made by HIV positive mothers and cross-feeding under unknown HIV status. The ability to prepare and administer the milk powder and to prepare and administer complementary feeding evidences the vulnerability of this population. Health professionals should ensure timely and adequate guidance to the family's understanding of the risk of vertical transmission. In order to ensure compliance with prophylaxis and safe and adequate feeding for non-breastfed infants, providing them with knowledge and skills in the daily care at home. Consequently, minimising their vulnerabilities, since parents are co-responsible for the health of their children. The family needs health education actions in an ongoing way to develop the daily care of the child.

\section{P4.58 ABSTRACT WITHDRAWN}

\section{P4.59 ONLINE VERSUS IN-PERSON TESTING: A QUALITATIVE ANALYSIS OF TESTING PREFERENCES AMONG YOUTH AND MEN WHO HAVE SEX WITH MEN USING AN ONLINE HIV/STI TESTING SERVICE IN VANCOUVER, CANADA}

${ }^{1}$ Mark Gilbert, ${ }^{1}$ Kimberly Thomson, ${ }^{2}$ Cathy Chabot, 'Devon Haag, ${ }^{3}$ Jean Shoveller. ${ }^{1}$ British Columbia Centre for Disease Control, Vancouver, Canada; ' University of British Columbia, Vancouver, Canada; ${ }^{3}$ British Columbia Centre for Excellence in HIVIAIDS, Vancouver, Canada

\subsection{6/sextrans-2017-053264.555}

Introduction Youth and men who have sex with men (MSM) are two priority populations with regards to sexual risk, HIV/ STI prevalence, and barriers to sexual healthcare. In 2014, an online HIV/STI testing service called GetCheckedOnline (GCO) was implemented in Vancouver, Canada to address several barriers to testing. We investigated the acceptability and perceptions of GCO among youth and MSM, and identified how various social positions related to age, sexual identity, and geography affected preferences for online versus in-person testing.

Methods We conducted in-depth, semi-structured interviews with 12 youth (ages 23-29) and 19 MSM (ages 30-71) who had used GCO at least once. Interviews were analysed for emergent themes and participants' sociodemographic data were collected via a brief questionnaire.
Results Youth participants identified predominantly as male (92\%), Caucasian (58\%), and heterosexual (50\%). MSM participants identified as male (100\%); Caucasian (84\%); and gay, bisexual, or pansexual $(68 \%, 26 \%, 5 \%)$. Both populations were motivated to use online testing for: convenience, not having to wait to get tested at a clinic, increased privacy/anonymity, and avoiding judgment from healthcare providers. Additionally, youth perceived online testing as modern and "the future." MSM participants perceived GCO as providing increased control over tests ordered and decreased anxiety due to receiving results faster. For three rurally-based MSM, GCO offered a way to test discreetly without identifying one's sexual orientation to a healthcare provider. Even among participants who reported routinely accessing face-to-face health services (including for health concerns other than STIs), GCO was described by most interviewees as advantageous in terms of convenience and privacy. Overall, $83 \%$ of youth and $84 \%$ of MSM said they would use GCO again.

Conclusion GCO was regarded as an acceptable and preferred option for accessing testing. Convenience was the most common reason for wanting to test online, although this varied somewhat by age, sexual orientation, and geography.

\section{P4.60 HOW DOES SEXUAL HEALTH CLINIC ATTENDANCE RELATE TO RISK BEHAVIOUR? FINDINGS FROM BRITAIN'S THIRD NATIONAL SURVEY OF SEXUAL ATTITUDES AND LIFESTYLES (NATSAL-3)}

${ }^{1}$ Mercer Ch, ${ }^{2}$ Rebecca Geary, ${ }^{1}$ Clare Tanton, ${ }^{1}$ Soazig Clifton, ${ }^{1}$ Fiona Mapp, ${ }^{3}$ Jackie Cassell, ${ }^{1}$ Pam Sonnenberg. ' UCL, London, UK; ${ }^{2}$ LSHTM, London, UK; ${ }^{3}$ Brighton and Sussex Medical School, Brighton, UK

\section{$10.1136 /$ sextrans-2017-053264.556}

Introduction In Britain, sexual health clinics (SHC) attendance has increased in recent decades. SHC remain the commonest place of STI diagnosis but many with STI risk behaviours do not attend. We explored attendance patterns and service preferences in those reporting 'unsafe sex' (condomless first sex with new partner and/or $\geq 2$ partners with no condom use, past year).

Methods Complex survey analyses of Natsal-3, a probability survey of 15162 people aged 16-74 years (6293 men), undertaken 2010-2012 using computer-assisted personal- and self-interviewing (CAPI/CASI).

Results Overall, recent SHC attendance (past year) was highest among those aged 16-24y (16.6\% men, 22.4\% women) and decreased with age $(<1.5 \%$ among those aged 45-74y). Approximately $18 \%$ of those $16-44 y \quad(n=771$ men; $n=1080$ women) reported unsafe sex; of these, $>75 \%$ had not attended a SHC in the past year. Among those reporting unsafe sex, non-attenders were older, and less likely to report $>2$ partners and/or concurrent partners (past year). Most of these non-attenders did not report chlamydia $(73 \%$ men, $41 \%$ women aged $<25 ; 86 \%$ men, $73 \%$ women aged $25+$ ) or HIV (97\% men, 93\% women) testing elsewhere (past year). The majority reporting unsafe sex who had previously attended a SHC would seek STI care there $(72 \%$ men, $66 \%$ women), whereas most who had not would go to general practice (66\% men, $77 \%$ women).

Conclusion While SHC attendance was more likely among those reporting STI risk behaviour, many reporting unsafe sex had not attended and most of these did not report chlamydia or HIV tests, indicating they are not receiving sexual 
healthcare elsewhere. This is of more concern for those at higher STI risk for example the younger, MSM and those with concurrent partners, although higher proportions of younger people reported Chlamydia testing elsewhere. We used unsafe sex as one measure of exposure to risk, which does not take into account characteristics of partner(s) and the wider sexual network. Further improvements are necessary to reach those at-risk, including through effective, diverse service provision.

\section{P4.61 SEXUAL IDENTITY, ATTRACTION AND EXPERIENCE IN BRITAIN: THE IMPLICATIONS OF USING DIFFERENT DIMENSIONS OF SEXUAL ORIENTATION TO ESTIMATE THE SIZE OF SEXUAL MINORITY POPULATIONS}

${ }^{1}$ Mercer Ch, ${ }^{2} \mathrm{R}$ Geary, ${ }^{1} \mathrm{C}$ Tanton, ${ }^{2} \mathrm{~B}$ Erens, ${ }^{1} \mathrm{~S}$ Clifton, ${ }^{3}$ Mitchell $\mathrm{Kr},{ }^{1} \mathrm{P}$ Sonnenberg. ${ }^{1} \mathrm{UCL}$, London, UK; ${ }^{2}$ LSHTM, London, UK; ${ }^{3}$ University of Glasgow, Glasgow, UK

\subsection{6/sextrans-2017-053264.557}

Introduction Sexual orientation encompasses three dimensions: sexual identity, attraction and behaviour. Many health and policy surveys and inequality monitoring activities collect data only on identity, while STI risk is primarily driven by behaviour. We present estimates of all three dimensions and examine the extent of their overlap, for men and women, and consider the implications of using the different criteria to estimate the size of sexual minority populations in Britain.

Methods Descriptive analyses of data from Britain's third National Survey of Sexual Attitudes and Lifestyles, a probability survey (15 162 people aged 16-74 years) undertaken 2010-2012 using a computer-assisted personal- and self-interviewing (CAPI/CASI). A standard question was used to define sexual identity (CAPI). Participants were also asked to describe their sexual attraction according to a scale ranging from exclusively opposite-sex to exclusively same-sex (CAPI). Same-sex sex was defined as ever having had sex (including genital contact) with someone of the same sex (CASI).

Results A lesbian, gay or bisexual (LGB) identity was reported by $2.5 \%$ of men and $2.4 \%$ of women, whilst $6.5 \%$ of men and $11.5 \%$ of women reported any same-sex attraction, and $5.5 \%$ of men and $6.1 \%$ of women reported ever experience of same-sex sex. This equates to approximately 573000 men and 559000 women in Britain currently self-identifying as LGB, less than half the number who are estimated to have ever had same-sex sex: 1,262,000 men and 1,422,000 women. Of those reporting having same-sex sex in the past 5 years, $28 \%$ of men and $45 \%$ of women identified as heterosexual.

Conclusions Substantial incongruity exists between the three measures of sexual orientation on an individual level, particularly for women. The size of sexual minority populations will depend on the dimension of sexual orientation applied, the choice of which depends on context and purpose. Regardless, the decision to use a particular dimension should be made explicitly, with a clear rationale, and with awareness of the limitations of each.

\section{P4.62 HIV TESTING PREFERENCES AMONG LONG DISTANCE TRUCK DRIVERS IN KENYA: A DISCRETE CHOICE EXPERIMENT}

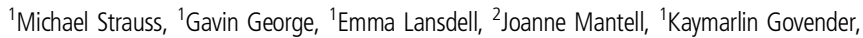
${ }^{3}$ Matthew Romo, ${ }^{4}$ Jacob Odhiambo, ${ }^{4}$ Eva Mwai, ${ }^{4}$ Eston Nyaga, ${ }^{3}$ Elizabeth Kelvin. ${ }^{1}$ Health Economics and HIV and AIDS Research Division, University of Kwazulu-Natal, Durban, South African Republic; ${ }^{2}$ HIV Centre for Clinical and Behavioural Studies, Department of Psychiatry, Division of Gender, New York, USA; ${ }^{3}$ Department of Epidemiology and Biostatistics, Cuny Graduate School of Public Health and Health Poli, New York, USA; ${ }^{4}$ North Star Alliance, Nairobi, Kenya

\subsection{6/sextrans-2017-053264.558}

Introduction Providing HIV testing to truck drivers in Africa is crucial but challenging. The introduction of HIV self-testing provides expanded service delivery options for clients, potentially increasing demand for services and expanding coverage - especially important for high-risk and difficult-to-reach populations. This study examines the preferences regarding HIV testing service delivery models, among long distance truck drivers in an effort to identify testing services that would appeal to this population.

Methods Using a discrete choice experiment, this study examines the drivers of choice regarding HIV counselling and testing among 305 truck drivers recruited from two roadside wellness clinics along major trucking routes in Kenya. Participants made trade-offs between characteristics of HIV testing service delivery models by making hypothetical choices in a series of paired HIV testing scenarios. Conditional logit models were used to identify the HIV testing characteristics driving the selection of preferred scenarios, and determine whether preferences interact with individual characteristics especially HIV testing history.

Results Participants preferred free, provider-administered HIV testing at a roadside clinic, using a finger-prick test, with inperson counselling, undertaken in the shortest possible time. The strongest driver of choice was the cost of the test. Those who had never tested previously preferred oral testing and telephonic counselling, while those who were not regular testers favoured clinic based- over self-testing.

Conclusion The results of this study indicate that for the majority of participants - most of whom had tested before the existing services offered at roadside clinics were the preferred service delivery model. The introduction of oral selftesting increases the options available to truck drivers and may even improve testing uptake for some, especially among those who have never tested before. However, these findings suggest the impact on HIV testing uptake of introducing oral self-testing may be limited in this population.

\section{P4.63 DESIGNING HUMAN IMMUNODEFICIENCY VIRUS COUNSELLING AND TESTING SERVICES TO MAXIMISE UPTAKE AMONG HIGH SCHOOL LEARNERS IN SOUTH AFRICA: WHAT MATTERS?}

${ }^{1}$ Michael Strauss, ${ }^{1}$ Gavin George, ${ }^{2}$ Bruce Rhodes. ${ }^{1}$ Health Economics and HIV and AIDS Research Division, University of Kwazulu-Natal, Durban, South African Republic; ${ }^{2}$ School of Accounting, Economics and Finance, University of Kwazulu-Natal, Durban, South African Republic

10.1136/sextrans-2017-053264.559 